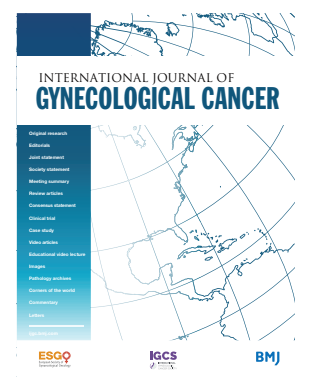

\title{
Outcomes of women treated with progestin and metformin for atypical endometrial hyperplasia and early endometrial cancer: a systematic review and meta-analysis
}

Jennifer Chae-Kim, ${ }^{1,2}$ Gunjal Garg, ${ }^{3}$ Larisa Gavrilova-Jordan, ${ }^{4}$ Lindsay E Blake, ${ }^{5}$ Tongil "TI" Kim, ${ }^{6}$ Qiang Wu, ${ }^{7}$ Clifford C Hayslip ${ }^{8}$

- Additional supplemental material is published online only. To view, please visit the journal online (http://dx.doi.org/ 10.1136/ijgc-2021-002699).

For numbered affiliations see end of article.

\section{Correspondence to}

Dr Jennifer Chae-Kim, Department of Obstetrics and Gynecology, Baylor Scott \& White Medical Center Temple, Temple, Texas, USA; jennifer. chaekim@bswhealth.org

Received 14 April 2021 Accepted 6 October 2021 Published Online First 16 November 2021

\section{Sinked}

- http://dx.doi.org/10.1136/ ijgc-2021-003192

Check for updates

(C) IGCS and ESG0 2021. No commercial re-use. See rights and permissions. Published by BMJ.

\begin{tabular}{l}
\hline To cite: Chae-Kim J, \\
Garg G, Gavrilova-Jordan L, \\
et al. Int J Gynecol Cancer \\
2021;31:1499-1505. \\
\hline
\end{tabular}

\section{HIGHLIGHTS}

- Women who receive progestin and metformin therapy have lower disease relapse than those receiving progestin alone.

- Combined therapy was not associated with significantly different remission rates.

- Combined therapy was not associated with significantly different pregnancy and live birth rates.

\section{ABSTRACT}

Objective Progestin therapy is the recommended fertility-sparing management of atypical endometrial hyperplasia or early-stage endometrial cancer in reproductive-aged women. Our objective was to evaluate disease relapse after progestin and metformin versus progestin therapy alone in patients with endometrial hyperplasia or cancer. Our secondary outcomes were disease remission, clinical pregnancy and live birth rate. Methods A systematic review of the literature was conducted (MEDLINE, Web of Science, Cochrane Library, CINAHL, LILACS, clinicaltrials.gov) from inception to April 2021. Studies of reproductive-aged women with atypical endometrial hyperplasia or early endometrial cancer who received progestin and metformin or progestin alone for fertility-sparing management, were included in the review. Early endometrial cancer was defined as grade 1, stage 1 disease. Exclusion criteria included women with higher grade endometrial cancer and when conservative management was not for fertility-sparing purposes. Data are presented as odds ratios (ORs) and 95\% confidence intervals (Cls) with fixed or random effects meta-analysis. Quality scoring was based on the Newcastle-Ottawa and Jadad scales.

Results In total, 271 reports were identified and six studies met the inclusion criteria. These studies included 621 women; 241 (38.8\%) patients received combined therapy and $380(61.2 \%)$ received progestin therapy alone. Relapse rates were lower for progestin and metformin than for progestin therapy alone (pooled OR $0.46,95 \% \mathrm{Cl} 0.24$ to $0.91, p=0.03$ ). The remission rates were not different (pooled OR 1.35, 95\% Cl 0.91 to 2.00, $\mathrm{p}=0.14$ ). Women who received progestin and metformin achieved pregnancy and live birth rates similar to those who received progestin therapy only (pooled OR 1.01, 95\% $\mathrm{Cl} 0.44$ to 2.35 , $\mathrm{p}=0.98$; pooled OR $0.46,95 \% \mathrm{Cl} 0.21$ to $1.03, \mathrm{p}=0.06$ ). Conclusion For reproductive-aged women with atypical endometrial hyperplasia or early endometrial cancer, progestin and metformin therapy compared with progestin therapy alone is associated with lower relapse rates, and similar remission, clinical pregnancy and live birth rates.
PROSPERO registration number CRD42020179069. disease remission,

\section{INTRODUCTION}

Endometrial cancer is the most common gynecologic malignancy in the United States, with nearly 66570 new cases diagnosed in 2021, and its incidence has been rising. ${ }^{1}$ Chronic exposure of the endometrium to unopposed estrogen, seen in chronic anovulation and obesity, predisposes women to the development of atypical endometrial hyperplasia and type 1 endometrial cancer. ${ }^{2}{ }^{3}$ With the increasing incidence of reproductive-aged women requiring fertility-sparing management of endometrial hyperplasia or cancer, conservative therapies have become accepted alternatives. ${ }^{4}$ Multiple studies have shown that women with early stage disease can be managed with oral progestin or levonorgestrel intrauterine device, ${ }^{4}$ which are now recommended fertility-sparing treatments. Criteria for fertility-sparing management of endometrial cancer and atypical endometrial hyperplasia include the following: pathology not worse than well differentiated (grade 1) endometrioid adenocarcinoma confirmed on biopsy; disease limited to the endometrium based on imaging; absence of metastatic disease; and no contraindications to medical therapy or pregnancy. ${ }^{5}$

In meta-analyses that examined conservative management of atypical endometrial hyperplasia or early endometrial cancer with progestin therapy, remission rates were as high as $76 \%$ for endometrial cancer, $85 \%$ for atypical endometrial hyperplasia, and live birth rates ranged from $26 \%$ to $41 \% .{ }^{467}$ Response to progestin therapy, however, may not be definitive because many patients have disease recurrence, with rates ranging from $35 \%-40 \%$ for endometrial cancer and $25 \%$ for atypical endometrial hyperplasia. ${ }^{6}$ These rates are concerning because these women delay 


\section{Original research}

definitive therapy in the hope of having children, and they bring attention to the need for a new therapeutic strategy. ${ }^{8}$

Metformin, a biguanide antidiabetic agent, has recently been repurposed for the management of endometrial cancer because of its anti-proliferative effects on endometrial cells. Metformin has also been shown to have a synergistic impact on progesterone's inhibitory effect on endometrial cell proliferation. ${ }^{9-11}$ Growing evidence in the literature suggests that metformin may be a beneficial adjunctive therapy, with a synergistic effect alongside progestin, in the suppression of endometrial proliferation.

A 2017 Cochrane systematic review reported on three studies that studied the effects of metformin on endometrial hyperplasia; only one of these studies compared the effects of progestin and metformin with progestin alone. ${ }^{12}$ Since then, additional studies have been added to the literature, and have also examined outcomes for patients with atypical hyperplasia and those with endometrial carcinoma. Although atypical endometrial hyperplasia is a pre-cancerous disease state, there is significant overlap between patients with atypical hyperplasia and those found to have invasive carcinoma after hysterectomy. Given this risk of underlying undiagnosed malignancy, or progression to cancer, the standard of care for both subsets of patients is hysterectomy. Additionally, both subsets of patients may be eligible for fertility-sparing management. The aim of this study was to conduct an updated systematic review of outcomes for fertility-sparing management of atypical endometrial hyperplasia and early endometrial cancer with progestin and metformin ('combined') versus progestin therapy alone.

\section{METHODS}

\section{Search Strategy}

We followed updated PRISMA guidelines for systematic reviews. ${ }^{13}$ A literature search was conducted by a medical librarian (LEB) identifying studies in PubMed MEDLINE, Web of Science, Cochrane Library, CINAHL, LILACS, and clinicaltrials.gov. These databases were searched from inception to April 2020 using key terms and subject headings when available. The same search was updated in April 2021. The full search strategy for PubMed is presented in the Online supplemental table 1 . References of primary and review articles were hand-searched to identify any other relevant studies not captured by the database search, however no new additional studies were included. Unpublished and gray literature were not included in the literature review. The primary outcome of interest was disease relapse. Secondary outcomes included disease remission, clinical pregnancy, and live birth rate.

\section{Study Selection}

Studies were selected if they met the inclusion criteria as follows: reproductive-aged women with a histologically confirmed diagnosis of atypical endometrial hyperplasia or early endometrial cancer, who met criteria for fertility-sparing management, and received fertilitysparing management with combined therapy or progestin therapy. Studies reporting on at least one of the outcomes were included in the meta-analysis. Relevant exclusion criteria included women with endometrial cancer classified as grade 2 or worse, and when conservative management was pursued due to poor surgical candidacy or perioperative morbidity. Interventional randomized or nonrandomized trials and observational prospective or retrospective studies were included. Case reports or series, editorial letters, and conference presentations were excluded from the analysis. If part of the study population met the inclusion criteria, however, patientlevel data could not be distinguished from those who did not meet the inclusion criteria after full-text analysis or correspondence with the senior author, the study was excluded from the meta-analysis. The complete review protocol was registered with PROSPERO under the registration number CRD42020179069 and can be accessed online at https://wwwcrdyorkacuk/prospero/.

\section{Data Extraction}

Studies were selected and screened by two reviewers independently (JC-K and GG), with a third reviewer (CCH) designated as a final consultant for any disagreements. The records were screened based on titles and abstracts. Full-text articles were then assessed for eligibility based on inclusion and exclusion criteria by the authors listed above. Extracted data, if available, included age, reported patient characteristics such as body mass index, race or ethnicity, medical history, histological diagnosis, primary and secondary outcomes. Data were kept on a Google spreadsheet. Inquiries regarding missing data were sent to corresponding authors. Yang and colleagues provided updated data regarding live birth outcomes, ${ }^{14}$ and these were incorporated into our analysis. Data that could not be retrieved after contacting authors on two separate occasions were not included in the analysis. In accordance with the journal's guidelines, we will provide our data for the reproducibility of this study in other centers if such requested.

\section{Quality Assessment of the Studies}

Study quality was evaluated using the Newcastle-0ttawa Scale ${ }^{15}$ for non-randomized studies, and the Jadad scale ${ }^{16}$ for randomized trials. Based on the Newcastle-0ttawa scale, we assigned 0-9 points to three criteria: selection, comparability, and outcome assessment. Based on the Jadad scale for randomized studies, we assigned 0-5 points to three criteria: randomization, blinding, and an account of all patients.

\section{Statistical Analysis}

Data were analyzed independently by two authors (TK and QW) using Stata 14.0 and $\mathrm{R}$ statistical computing software, respectively. A random-effects meta-analysis model was selected to evaluate the pooled OR of primary outcomes when there was statistical heterogeneity in at least five unique studies. Otherwise, the fixed-effect model was employed. Data are presented as odds ratio (ORs) with $95 \%$ confidence intervals (Cls). A P value $<0.05$ was considered to indicate statistical significance. Subgroup analysis by study type, comparing randomized trials with retrospective studies, was pursued as well.

\section{RESULTS}

\section{Study Characteristics}

The initial literature search yielded 251 reports, and the updated search in April 2021 yielded an additional 20 reports, making a total of 271 reports. No additional studies were identified after review of the reference lists of primary and review articles. After removing duplicate records and an initial screen of citations and abstracts, 206 articles were excluded and 54 full-text articles were examined. Ultimately, six studies met the inclusion criteria for 


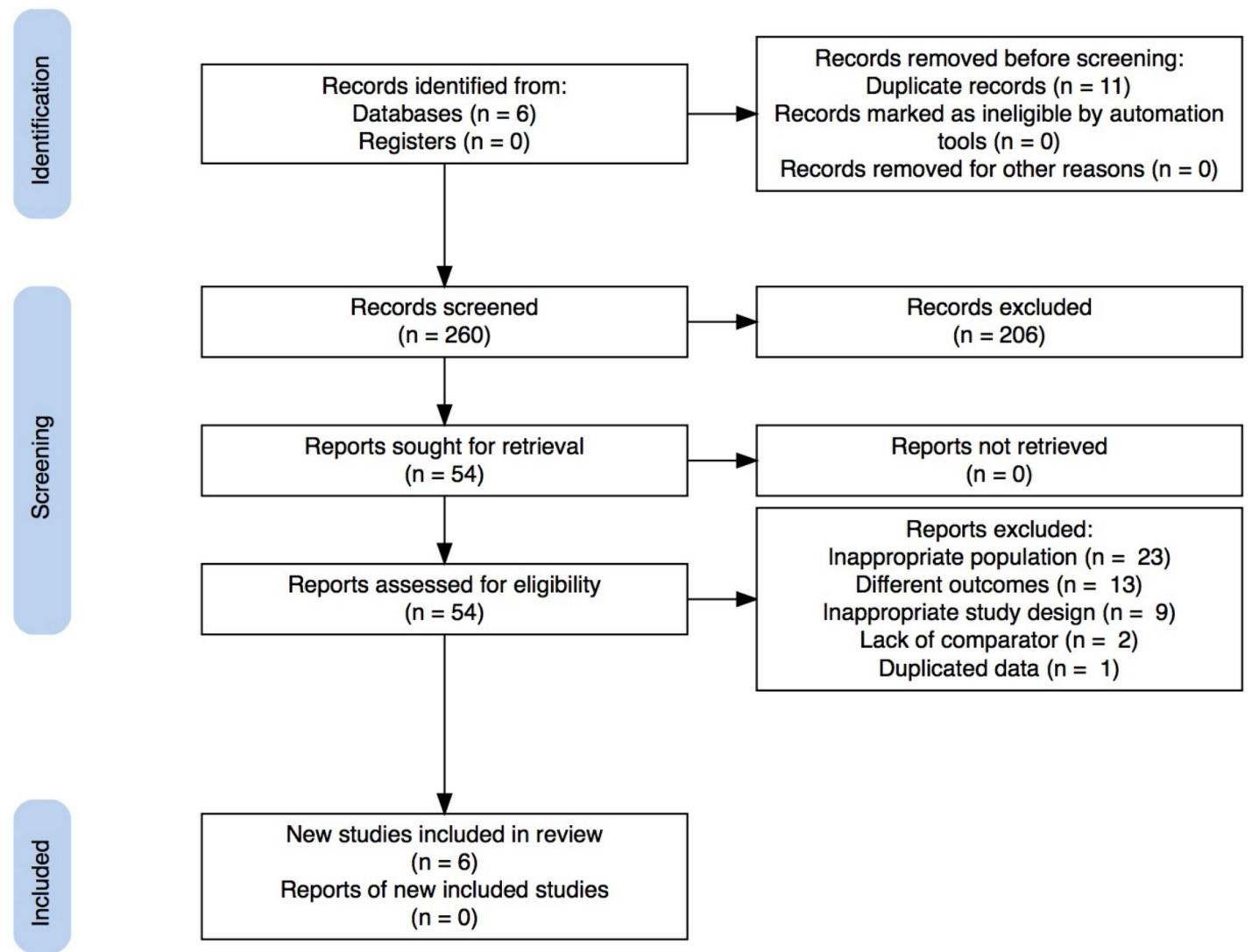

Figure 1 PRISMA flow diagram.

meta-analysis. ${ }^{14-21}$ The PRISMA flow chart describing the selection process is presented in Figure 1.

These six studies reported data on 621 women; 241 (38.8\%) patients who received combined therapy, and $380(61.2 \%)$ patients who received progestin therapy alone. Four studies were retrospective ${ }^{17-1921}$ and two were randomized clinical trials. ${ }^{1420}$ For all studies, the diagnosis of atypical endometrial hyperplasia or endometrial carcinoma was histologically determined. Most of the study population, specifically $472(76 \%)$ women, had been diagnosed with atypical endometrial hyperplasia, and 149 (24\%) women had endometrial cancer. Studies did not consistently distinguish between these groups of patients when reporting data, and did not report outcomes in a diagnosis-specific manner.

Mean age and body mass index for the combined therapy group were 33.8 years and $31.9 \mathrm{~kg} / \mathrm{m}^{2}$ compared with 34.6 years and $27.5 \mathrm{~kg} / \mathrm{m}^{2}$ in the progestin group. Studies did not uniformly report summary statistics and SD could not be calculated for age or body mass index. Both combined therapy and progestin therapy alone groups had a high percentage of nulliparous women at $78.9 \%$ and $78.3 \%$, respectively. The duration of metformin therapy was reported by three studies ${ }^{141718}$ and ranged from 6 months ${ }^{14}$ to 18 months, ${ }^{17}$ or until conception or disease recurrence. ${ }^{18}$ Three studies reported adverse effects of treatment, ${ }^{14} 1820$ noting the most common adverse effects of metformin were nausea or diarrhea, and that few patients discontinued therapy due to adverse effects. Two studies reported hormone receptor status ${ }^{1920}$; in one study, the histological evaluation of all patients in the study revealed positive estrogen and progesterone receptors ${ }^{19}$; another study reported minimal difference in expression of estrogen and progesterone receptors before and after therapy with either progestin or combined treatment. ${ }^{20}$ The main characteristics of the included studies are shown in Table 1.

\section{Quality Assessment}

For studies evaluated on the Newcastle-0ttawa Scale, the final quality assessment scores ranged from 7 to 8 , and the average score was 7.25. For studies evaluated on the Jadad scale, the assessment scores ranged from 3 to 4 , and the average score was 3.5 .

\section{Meta-analysis}

Primary Outcome: Relapse

A meta-analysis of three studies found that the odds of relapse were statistically lower for combined therapy compared with progestin therapy alone (pooled $\mathrm{OR} 0.46,95 \% \mathrm{Cl} 0.24$ to $0.91, \mathrm{p}=0.03$ ). Two of the studies were retrospective, ${ }^{1718}$ and one was a randomized trial. ${ }^{14}$ Subgroup analysis was performed for retrospective studies only, and the relapse rate was found to be statistically lower for combined therapy $(\mathrm{OR} 0.30,95 \% \mathrm{Cl} 0.13$ to $0.72, \mathrm{p}<0.01)$. See Figure 2 .

Secondary Outcomes: Remission, Clinical Pregnancy, Live Birth Rate

Meta-analysis of all six studies found that the OR for remission was not statistically different between combined and progestin 


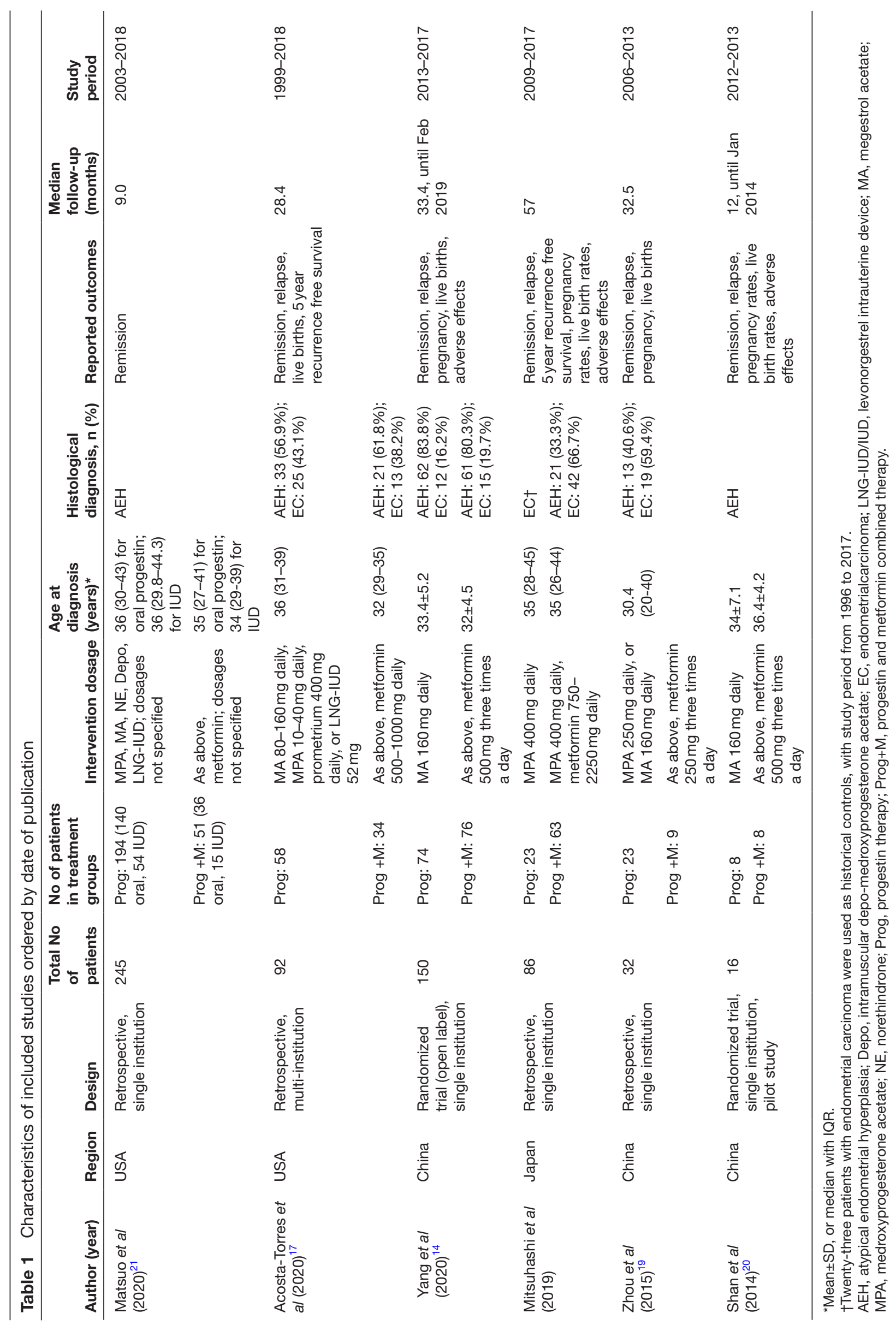


A Relapse

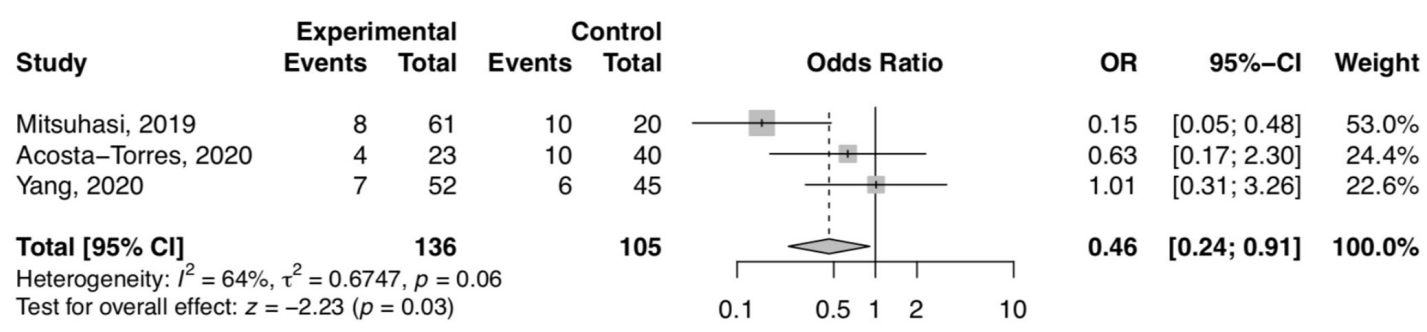

B Remission

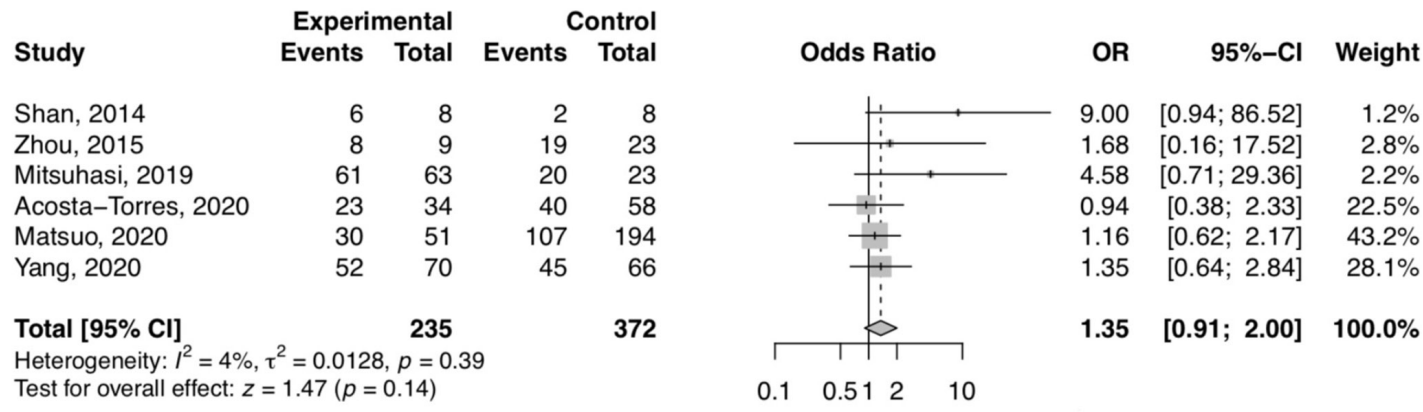

C Clinical pregnancy

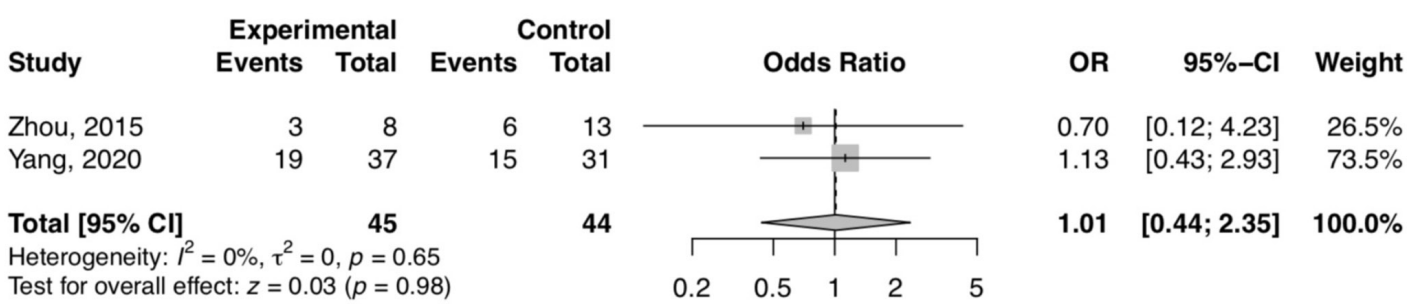

D Live birth rate

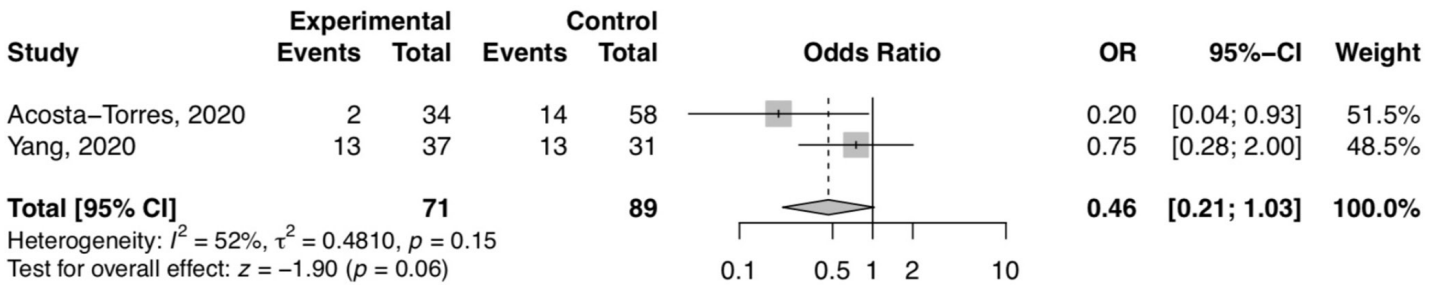

Figure 2 Odds ratios of outcomes.

therapy (pooled OR 1.35, 95\% Cl 0.91 to $2.00, \mathrm{p}=0.14$ ). A metaanalysis of two studies found the odds of clinical pregnancy for combined therapy did not significantly differ from that of progestin therapy alone $(\mathrm{OR} 1.01,95 \% \mathrm{Cl} 0.44$ to $2.35, \mathrm{p}=0.98)$. A meta-analysis of two studies found the odds of live birth for combined versus progestin therapy groups were not statistically different at the 0.05 level, although combined therapy was associated with a lower odds of live birth that was marginally significant at the 0.1 level (pooled $0 \mathrm{R} 0.46,95 \% \mathrm{Cl} 0.21$ to 1.03 , $\mathrm{p}=0.06$ ). Subgroup analysis of retrospective studies showed that the $\mathrm{OR}$ for remission was not significantly different between the two groups. Subgroup analysis of pregnancy and live birth rate outcomes was not meaningful due to the limited number of studies. 


\section{Original research}

\section{DISCUSSION}

\section{Summary of Main Results}

This study provides an overview of the current evidence on the outcomes of fertility-sparing therapy with progestin and metformin versus progestin alone for atypical endometrial hyperplasia and early endometrial cancer in reproductive-aged women. While remission rates were not significantly different, relapse rates were statistically lower for women who received combined therapy than those who received progestin therapy alone. Women who received progestin and metformin achieved pregnancy and live birth rates similar to those who received progestin only.

\section{Results in the Context of Published Literature}

Investigation of this novel treatment strategy has clinical relevance with the rising incidence of endometrial cancer in young, reproductive-aged women who require fertility-sparing treatment. Conservative management involves progestin therapy, which has been shown in many studies to be associated with high recurrence or relapse rates, reported around $25 \%-40 \% .{ }^{46}$ Our meta-analysis of relapse rates in women who received combined therapy were significantly lower compared with those who received progestin therapy only. Metformin has been shown to modulate expression of steroid receptors, having the effect of decreasing estrogen receptor and increasing progesterone receptor expression. ${ }^{22}$ Some elements of progesterone resistance may be seen in endometrial cancer cells with progestin therapy, which may explain the high relapse rates found in clinical studies. If progesterone resistance is due to downregulation of progesterone receptor expression, metforminmediated upregulation of progesterone receptor may contribute to our findings of significantly lower relapse rates.

Our study did not find significant differences in reproductive outcomes of clinical pregnancy or live birth rate based on a limited number of studies. Combined progestin and metformin therapy was associated with a lower live birth rate that was marginally significant, indicating the need for further research. It was difficult to ascertain from the available data whether patients receiving metformin were more likely to have infertility, or some other underlying pathology that may contribute to infertility. Matsuo and colleagues examined characteristics associated with metformin use and found that women who received metformin were in fact more likely to have diabetes, hyperlipidemia or polycystic ovary syndrome. ${ }^{21}$ Further, given that women with polycystic ovary syndrome ${ }^{23}$ or diabetes mellitus ${ }^{2425}$ are more likely to develop atypical endometrial hyperplasia or endometrial cancer, there may have been selection bias in the study population.

While the clinical pregnancy rates of the two groups were not significantly different, the pregnancy rates for our study (54\% for progestin and metformin combined therapy, $48 \%$ for progestin therapy alone) appear higher than what has been previously reported (approximately 34\%-41\%). ${ }^{4}$ We suspect this difference may be due to higher utilization of assisted reproductive technology. Not all studies reported on assisted reproduction versus spontaneous pregnancy, however it appears a vast majority of study participants pursued assisted reproduction. Two studies ${ }^{17}{ }^{19}$ reported the proportion of clinical pregnancies that required assisted reproduction, and this ranged from $81 \%$ to $89 \%$. This proportion is substantially higher than the $54.1 \%$ of women who achieved clinical pregnancy by way of assisted reproductive technology, as reported in an earlier systematic review. ${ }^{3}$ Assisted reproductive technology is often recommended for patients undergoing fertility-sparing therapy, given the risk of relapse. Our findings of lower relapse rates for women on combined progestin and metformin therapy suggest that this therapy may be beneficial and should be considered for patients who face barriers to timely entry to infertility care.

\section{Strengths and Weaknesses}

The main strength of this study is that it is the first systematic review and meta-analysis of this topic that examines outcomes after progestin and metformin therapy for patients with endometrial hyperplasia and cancer. This review provides insight into outcomes related to a new treatment regimen and identifies areas for future research. There are a number of limitations to this meta-analysis. First, there were a limited number of studies meeting the inclusion criteria. Many of the included studies reported outcomes for both atypical endometrial hyperplasia and endometrial carcinoma, without distinguishing between these groups, therefore the metaanalysis reports outcomes for these patients together. Further, a majority of the included patients had atypical endometrial hyperplasia, and this may skew the results. There may be a number of confounding factors such as age, race or ethnicity, body mass index, or baseline fertility status. We were unable to statistically compare the body mass indices of women who received combined versus progestin therapy alone due to missing data in multiple studies. From the available data, however, the average body mass index for women who received combined therapy appears higher $\left(31.9 \mathrm{~kg} / \mathrm{m}^{2}\right)$ than that of those who received progestin therapy only $\left(27.5 \mathrm{~kg} / \mathrm{m}^{2}\right)$. In light of research indicating that patients with endometrial cancer with higher body mass index have poorer outcomes including relapse, ${ }^{26}$ the decreased relapse rates seen in the combined therapy group is encouraging.

Other important limitations are variations in the administration of progestin and metformin. There was heterogeneity in the progestin type utilized in the included studies. One of the included studies compared systemic oral progestin with local progestin intrauterine device, and found that metformin was more effective with local progestin. ${ }^{21}$ Other research has shown no significant difference in endometrial hyperplasia or cancer response outcomes when comparing systemic versus local effects of progestin. ${ }^{27}$ The doses of metformin ranged from $500 \mathrm{mg}$ daily to $2250 \mathrm{mg}$ daily, or was unspecified. Only three studies reported on duration of metformin therapy. In one study, about a quarter of the patients who received combined therapy continued metformin for a mean of 18.3 months, ${ }^{17}$ and other studies reported that patients continued metformin for at least 6 months if they did not achieve complete remission, ${ }^{14}$ or until conception or disease recurrence. ${ }^{18}$ More studies are needed to determine the optimal window between discontinuation of combined progestin and metformin therapy and onset of pregnancy.

\section{Implications for Practice and Future Research}

The implications of our findings for clinical practice are still uncertain. Further prospective, randomized investigation is needed to overcome the limitations of this study.

\section{CONCLUSIONS}

In conclusion, this meta-analysis shows that combined progestin and metformin therapy is associated with decreased disease 
relapse rates. Disease remission, clinical pregnancy, and live birth rates were similar for patients who received progestin and metformin versus progestin therapy. A review of the literature included a limited number of moderate quality studies, indicating the need for further research.

\section{Author affiliations}

${ }^{1}$ Department of Obstetrics and Gynecology, East Carolina University Brody School of Medicine, Greenville, North Carolina, USA

${ }^{2}$ Department of Obstetrics and Gynecology, Baylor Scott \& White Medical Center Temple, Temple, Texas, USA

${ }^{3}$ Division of Gynecologic Oncology, East Carolina University Brody School of Medicine, Greenville, North Carolina, USA

${ }^{4}$ Division of Reproductive Endocrinology, Infertility and Genetics, Augusta University Medical College of Georgia, Augusta, Georgia, USA

${ }^{5}$ University of Arkansas for Medical Sciences Library, University of Arkansas for Medical Sciences, Little Rock, Arkansas, USA

${ }^{6}$ Department of Marketing, Naveen Jindal School of Management, The University of Texas at Dallas, Richardson, Texas, USA

${ }^{7}$ Department of Biostatistics, East Carolina University, Greenville, North Carolina, USA

${ }^{8}$ Division of Reproductive Endocrinology and Infertility, East Carolina University Brody School of Medicine, Greenville, North Carolina, USA

Acknowledgements The authors would like to acknowledge the Departments of Obstetrics and Gynecology at Texas A\&M College of Medicine, at Baylor Scott \& White Health, and Brody School of Medicine at East Carolina University, and Vidant Medical Center for supporting this research.

Contributors JC-K: conceptualization, methodology, validation, investigation, writing - original draft, writing - review \& editing, visualization, project administration, guarantor. GG: methodology, verification, writing - review \& editing, visualization, supervision. LG-J: writing - review \& editing, visualization, supervision. LEB: methodology, investigation, resources, data curation. TK: methodology, software, formal analysis. QW: methodology, software, formal analysis. CCH: writing - review \& editing, visualization, supervision.

Funding The authors have not declared a specific grant for this research from any funding agency in the public, commercial or not-for-profit sectors.

Competing interests None declared.

Patient consent for publication Not applicable.

Provenance and peer review Not commissioned; externally peer reviewed.

Data availability statement Data are available upon reasonable request.

Supplemental material This content has been supplied by the author(s). It has not been vetted by BMJ Publishing Group Limited (BMJ) and may not have been peer-reviewed. Any opinions or recommendations discussed are solely those of the author(s) and are not endorsed by BMJ. BMJ disclaims all liability and responsibility arising from any reliance placed on the content. Where the content includes any translated material, BMJ does not warrant the accuracy and reliability of the translations (including but not limited to local regulations, clinical guidelines, terminology, drug names and drug dosages), and is not responsible for any error and/or omissions arising from translation and adaptation or otherwise.

\section{REFERENCES}

1 American Cancer Society. Cancer statistics center, 2021. Available: http://cancerstatisticscenter.cancer.org [Accessed 27 Sep 2021].

2 Hacker NF, Friedlander MLBerek JS, Hacker NF, eds. Gynecologic oncology. 7th edn. Philadelphia: Wolters Kluwer, 2021.

3 Koskas M, Uzan J, Luton D, et al. Prognostic factors of oncologic and reproductive outcomes in fertility-sparing management of endometrial atypical hyperplasia and adenocarcinoma: systematic review and meta-analysis. Fertil Steril 2014;101:785-94.

4 Gunderson CC, Fader AN, Carson KA, et al. Oncologic and reproductive outcomes with progestin therapy in women with endometrial hyperplasia and grade 1 adenocarcinoma: a systematic review. Gynecol Oncol 2012;125:477-82.
5 Koh W-J, Abu-Rustum NR, Bean S, et al. Uterine Neoplasms, Version 1.2018, NCCN Clinical Practice Guidelines in Oncology.. J Natl Compr Canc Netw 2018;16:170-99.

6 Gallos ID, Yap J, Rajkhowa M, et al. Regression, relapse, and live birth rates with fertility-sparing therapy for endometrial cancer and atypical complex endometrial hyperplasia: a systematic review and metaanalysis. Am J Obstet Gynecol 2012;207:266.e1-12.

7 Wei J, Zhang W, Feng L, et al. Comparison of fertility-sparing treatments in patients with early endometrial cancer and atypical complex hyperplasia: a meta-analysis and systematic review. Medicine 2017;96:e8034.

8 Banno K, lida M, Yanokura M, et al. Drug repositioning for gynecologic tumors: a new therapeutic strategy for cancer. ScientificWorldJournal 2015;2015:1-10.

9 Cao C, Zhou J-Y, Xie S-W, et al. Metformin enhances Nomegestrol acetate suppressing growth of endometrial cancer cells and may correlate to downregulating mTOR activity in vitro and in vivo. Int $J$ Mol Sci 2019;20:3308.

10 Jiang $\mathrm{Y}$, Chen X, Wei Y, et al. Metformin sensitizes endometrial cancer cells to progestin by targeting TET1 to downregulate glyoxalase I expression. Biomed Pharmacother 2019;113.

11 Mu N, Dong M, Li L. Synergistic effect of metformin and medroxyprogesterone 17-acetate on the development of endometrial cancer. Oncol Rep 2018;39:2015-21.

12 Clement NS, Oliver TRW, Shiwani H, et al. Metformin for endometrial hyperplasia. Cochrane Database Syst Rev 2017;2017.

13 Page MJ, McKenzie JE, Bossuyt PM, et al. The PRISMA 2020 statement: an updated guideline for reporting systematic reviews. BMJ 2021;372:n71.

14 Yang B-Y, Gulinazi Y, Du Y, et al. Metformin plus megestrol acetate compared with megestrol acetate alone as fertility-sparing treatment in patients with atypical endometrial hyperplasia and well-differentiated endometrial cancer: a randomised controlled trial. BJOG 2020;127:848-57.

15 Wells GA, Shea B, O'Connell D. The Newcastle-Ottawa scale (NOS) for assessing the quality of nonrandomised studies in meta-analyses. Available: http://www.ohri.ca/programs/clinical epidemiology/oxford.asp [Accessed 14 April 2021].

16 Jadad AR, Moore RA, Carroll D, et al. Assessing the quality of reports of randomized clinical trials: is blinding necessary? Control Clin Trials 1996;17:1-12.

17 Acosta-Torres S, Murdock T, Matsuno R, et al. The addition of metformin to progestin therapy in the fertility-sparing treatment of women with atypical hyperplasia/endometrial intraepithelial neoplasia or endometrial cancer: little impact on response and low live-birth rates. Gynecol Oncol 2020;157:348-56.

18 Mitsuhashi A, Habu Y, Kobayashi T, et al. Long-term outcomes of progestin plus metformin as a fertility-sparing treatment for atypica endometrial hyperplasia and endometrial cancer patients. J Gynecol Oncol 2019;30:1-11.

19 Zhou R, Yang Y, Lu Q, et al. Prognostic factors of oncological and reproductive outcomes in fertility-sparing treatment of complex atypical hyperplasia and low-grade endometrial cancer using ora progestin in Chinese patients. Gynecol Oncol 2015;139:424-8.

20 Shan W, Wang C, Zhang Z, et al. Conservative therapy with metformin plus megestrol acetate for endometrial atypical hyperplasia. J Gynecol Oncol 2014;25:214.

21 Matsuo K, Mandelbaum RS, Ciccone M, et al. Route-specific association of progestin therapy and concurrent metformin use in obese women with complex atypical hyperplasia. Int J Gynecol Cancer 2020;30:1-9.

22 Collins G, Mesiano S, DiFeo A. Effects of metformin on cellular proliferation and steroid hormone receptors in patient-derived, lowgrade endometrial cancer cell lines. Reprod Sci 2019;26:609-18.

23 Yin W, Falconer $\mathrm{H}$, Yin L, et al. Association between polycystic ovary syndrome and cancer risk. JAMA Oncol 2019;5.

24 Mitsuhashi A, Shozu M. New therapeutic approaches for the fertilitysparing treatment of endometrial cancer. J Obstet Gynaecol Res 2020:46:215-22.

25 Zhang Z-H, Su P-Y, Hao J-H, et al. The role of preexisting diabetes mellitus on incidence and mortality of endometrial cancer. Int'/ J Gynecol Can 2013;23:294-303

26 Schmandt RE, Iglesias DA, Co NN, et al. Understanding obesity and endometrial cancer risk: opportunities for prevention. Am J Obstet Gynecol 2011;205:518-25.

27 Hubbs JL, Saig RM, Abaid LN, et al. Systemic and local hormone therapy for endometrial hyperplasia and early adenocarcinoma. Obstet Gynecol 2013;121:1172-80. 